\title{
Asymptotes of multiplicity and transverse momentum cor- relation coefficients at large string density
}

\author{
Svetlana Belokurova ${ }^{1, *}$ \\ ${ }^{1}$ St Petersburg State University
}

\begin{abstract}
The multiple hadron production in high energy collisions is studied in the model with string fusion on transverse lattice.

In the framework of the model the asymptotes of the correlation coefficients between the mean transverse momentum and the multiplicity and between the mean transverse momenta for realistic case of non-uniform string distribution in transverse plane were calculated. The results were obtained by two different methods using the alternative definitions of the correlation coefficients. It is shown that the asymptotes of the correlation coefficients obtained by these two methods coincide in the leading approximation.

The examples with non-uniform string distribution in transverse plane were considered. Strong dependence of the correlation coefficient between the transverse momentum and a multiplicity on non-uniformity in the distribution of the strings was found. In particular it is shown that there are distributions of strings for which this coefficient becomes negative.

For the correlation coefficient between transverse momenta its dependence on the scaled variance of the number of particles produced from a single string, which disappears in the case of a homogeneous string distribution, is analyzed. The analytical asymptote for the correlation coefficient between transverse momenta obtained at large string density are compared with the results of the MC numerical calculations of this coefficient.
\end{abstract}

\section{Introduction}

Unfortunately at the present the quantum chromodynamics does not enable to describe numerically the soft part of multi-particle production. The different versions of the QCDinspired quark-gluon string model are used for a description of this component of hadronic interaction at high energy. One of the most popular approaches is the string model [1-4]. In this model at first stage the color quark-gluon strings are formed. At second stage the hadronization of these strings produces the observed hadrons.

In the case of ultrarelativistic heavy ion collisions it is more convenient to pass from the longitudinal component of particle momentum to the rapidity: $y=\frac{1}{2} \ln \frac{p_{0}+p_{z}}{p_{0}-p_{z}}$. One of the important consequences of the string model are correlations between multiplicities of charged particles in two rapidity windows ("forward" and "backward") separated by some gap - the long-range rapidity correlations, which were observed experimentally.

At present three types of correlation coefficients are usually studied: $n-n$, the correlation between multiplicities of charged-particles, $p_{t}-n$, the correlation between mean transverse

*e-mail: sveta.1596@mail.ru 


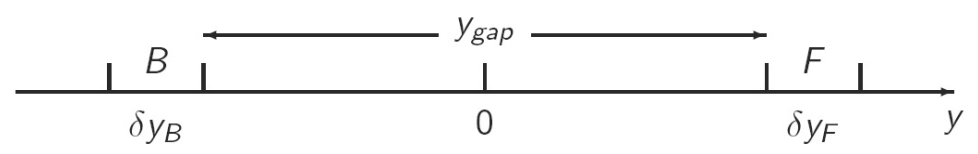

Figure 1. Forward "F" and backward "B" rapidity windows separated by a gap

momenta in one rapidity interval and the multiplicity of charged-particles in another interval, $p_{t}-p_{t}$, the correlation between values of mean transverse momenta. The multiplicity $n$ is an extensive variable, it depends on the volume of the system. Therefore, it is more promising to study the correlations involving the mean event momentum of produced particles, $p_{t}$, which is an intensive variable. It is sensitive to the fluctuation in the quality of sources, being not sensitive to the fluctuation in their number. In particular, this variable property can be used for the observation of the so-called string fusion phenomenon.

The string density in the transverse plane increases with the growth of initial energy or going to collisions of nuclei, and it is necessary also to take into account an interaction between the strings [5, 6], which leads to the formation of fused strings [7, 8].

To simplify the account of string fusion processes in our calculations we use the version of the model with the transverse lattice (grid) [9-11]. In the model the transverse plane is divided into cells, which area is equal to the string transverse cross-section. It is supposed that the strings with the centers in one cell are fused.

\section{Model}

Event is characterized by the set of configurations: $C=\left\{C_{\eta}, C_{n}^{B}, C_{n}^{F}, C_{p}^{B}, C_{p}^{F}\right\}$, where $C_{\eta}=\left\{\eta_{1}, \ldots \eta_{M}\right\}$ is string configuration, $\eta_{i}$ is the number of strings with centers in the $i$-th cell, $M$ is the number of cells in the transverse plane, $C_{n}^{F}=\left\{n_{1}^{F}, \ldots, n_{M}^{F}\right\}, C_{n}^{B}=$ $\left\{n_{1}^{B}, \ldots, n_{M}^{B}\right\}$ are the numbers of particles produced from the hadronizations of the string in forward and backward rapidity windows and $C_{p}^{F}=\left\{p_{1}^{1 F}, \ldots, p_{1}^{n_{1} F} ; \ldots ; p_{M}^{1 F}, \ldots, p_{M}^{n_{M} F}\right\}$, $C_{p}^{B}=\left\{p_{1}^{1 B}, \ldots, p_{1}^{n_{1} B} ; \ldots ; p_{M}^{1 B}, \ldots, p_{M}^{n_{M} B}\right\}$ are the transverse momentum of these particles.

In calculations the Gaussian approximation (formulated e.g. in [15]) was used: fluctuations in the number of strings in the cell are assumed independent and Gaussian distributed with variance proportional to the mean number of strings in the cell:

$$
\begin{gathered}
P\left(\eta_{i}\right)=\frac{1}{\sqrt{2 \pi d_{\eta_{i}}}} e^{-\frac{\left(\eta_{i}-\bar{\eta}_{i}\right)^{2}}{2 \eta_{i}}}, \\
d_{\eta_{i}}=\omega_{\eta} \bar{\eta}_{i},
\end{gathered}
$$

and similarly for the number of particles in forward and backward windows:

$$
\begin{gathered}
P\left(n_{i}^{F}\right)=\frac{1}{\sqrt{2 \pi d_{n_{i}^{F}}}} e^{-\frac{\left(n_{i}^{F}-n_{i}^{F}\right)^{2}}{2 n_{n_{i}^{F}}}}, P\left(n_{i}^{B}\right)=\frac{1}{\sqrt{2 \pi d_{n_{i}^{B}}}} e^{-\frac{\left(n_{i}^{B}-\bar{n}_{i}^{B}\right)^{2}}{2 n_{n_{i}^{B}}}}, \\
d_{n_{i}^{F}}=\omega_{\mu} \bar{n}_{i}^{F}, d_{n_{i}^{B}}=\omega_{\mu} \bar{n}_{i}^{B} .
\end{gathered}
$$

Also the average transverse momentum of the particles produced from the hadronization of the strings in the cells is assumed independent on the numbers of particles and depend on 
the numbers of strings in the cell. The variance of the transverse momentum of a particle is assumed proportional to the mean transverse momentum squared:

$$
d_{p_{i}}\left(\eta_{i}\right)=\overline{p^{2}}\left(\eta_{i}\right)-\bar{p}^{2}\left(\eta_{i}\right)=\gamma \bar{p}^{2}\left(\eta_{i}\right)
$$

In the framework of the string fusion model the dependence of the average number of particles and the transverse momentum of these particles formed from decay of the fused strings in the cell on the number of strings have the following form:

$$
\bar{n}\left(\eta_{i}\right)=\sqrt{\eta_{i}}, \bar{p}\left(\eta_{i}\right)=p_{0} \sqrt[4]{\eta_{i}} .
$$

The average numbers of particles formed from the hadronizations of the strings in i-th cell in forward rapidity window is assumed to be equal:

$$
\bar{n}_{i}^{F}=\mu_{F} \bar{n}\left(\eta_{i}\right)
$$

And the same for the backward window:

$$
\bar{n}_{i}^{B}=\mu_{B} \bar{n}\left(\eta_{i}\right)
$$

The asymptotes of the correlation coefficients were calculated supposing the existence of two small parameters:

$$
\frac{1}{\bar{\eta}_{i}} \ll 1, \frac{1}{M} \ll 1,
$$

where $M$ is a total number of cells in the transverse grid.

\section{Calculations}

Long-range rapidity is characterized by the correlation coefficient. There are two alternative definitions of correlation coefficient. The definitions generally are not equivalent for nonlinear regression function.

The strength of the long-range rapidity correlations is characterized by the correlation coefficient. There are two alternative definitions of a correlation coefficient. The first one is based on the covariance:

$$
b_{B F}^{\text {mean }} \equiv \frac{\langle F B\rangle-\langle F\rangle\langle B\rangle}{\left\langle F^{2}\right\rangle-\langle F\rangle^{2}},
$$

where $\langle F\rangle,\langle B\rangle$ is a mean values of $F$ and $B,\langle\ldots\rangle$ is averaging over all events. And the second one is based on the correlation (regression) function:

$$
\left.b_{B F}^{c o r r f} \equiv \frac{d\langle B\rangle_{F}}{d F}\right|_{F=\langle F\rangle},
$$

$\langle B\rangle_{F}$ is the correlation function.

Clearly that these two definitions generally (for nonlinear regression function) are not equivalent.

The both definitions were used in a number of papers [10, 12, 15-17].

In accordance with the first definition we need to calculate the covariance and the variance in the enumerator and the denominator of formula (10), averaging over all events with necessary accuracy. Averaging over events was performed in stages: firstly, the average values for 
a fixed string configuration were calculated; after this averaging over the string configuration was made.

$$
\langle F\rangle=\left\langle\left\langle\langle F\rangle^{C_{p}^{F}}\right\rangle^{C_{n}^{F}}\right\rangle^{C_{\eta}}
$$

where $\langle\cdots\rangle^{C}$ is averaging over the configuration $C$,

$$
\langle F\rangle^{C_{\eta}}=\prod_{i=1}^{M} \int d \eta_{i} P\left(\eta_{i}\right) F,\langle F\rangle^{C_{n}^{F}}=\prod_{i=1}^{M} \int d n_{i}^{F} P\left(n_{i}^{F}\right) F .
$$

According to the second definition, correlation coefficient is the correlation function derivative. To calculate the correlation function we have used the following representation from the paper [17]:

$$
\langle B\rangle_{F}=\frac{\sum_{C}\langle B\rangle_{C} P(C) P_{C}(F)}{\sum_{C} P(C) P_{C}(F)}
$$

In the case of large string density the configuration sum can be approximately rewritten as product of integrals:

$$
\begin{gathered}
\sum_{C_{\eta}} \ldots=\prod_{j=1}^{M} \sum_{\eta_{j}=0}^{\infty} \ldots \longrightarrow \prod_{j=1}^{M} \int_{0}^{\infty} d \eta_{j} \ldots, \\
\langle B\rangle_{F}=\frac{1}{P(F)} \prod_{j=1}^{M} \int_{0}^{\infty} d \eta_{j} P\left(C_{\eta_{j}}\right) \int_{0}^{\infty} d n_{j}^{B} P_{C_{\eta}}\left(C_{n}^{B}\right)\langle B\rangle_{C_{\eta} C_{n}^{B}} \int_{0}^{\infty} d n_{j}^{F} P_{C_{\eta}}\left(C_{n}^{F}\right) P_{C_{\eta} C_{n}^{F}}(F), \\
P(F)=\prod_{j=1}^{M} \int_{0}^{\infty} d \eta_{j} P\left(C_{\eta_{j}}\right) \int_{0}^{\infty} d n_{j}^{B} P_{C_{\eta}}\left(C_{n}^{B}\right) \int_{0}^{\infty} d n_{j}^{F} P_{C_{\eta}}\left(C_{n}^{F}\right) P_{C_{\eta} C_{n}^{F}}(F) .
\end{gathered}
$$

The asymptotes of the obtained integrals were found by the saddle-point method.

\section{Results}

In the model with string fusion the analytical expressions for the asymptotes of the longrange correlation coefficients for all three types of the correlations between different combinations of observables $\left(n-n, p_{t}-n, p_{t}-p_{t}\right)$ in these two observation windows separated in rapidity was founded. The asymptotes were calculated at large string density, arbitrary distributed in transverse plane, what makes the results of the calculations closer to the experimental situation.

The asymptotes were calculated by two different methods using the alternative definitions of the correlation coefficients. In the framework of our model the results occur coinciding for the different definitions.

$$
\begin{gathered}
b_{n n}^{\text {mean }}=b_{n n}^{\text {corr } f}=\frac{\omega_{\eta} \mu_{B} M}{4 \omega_{\mu} S_{1 / 2}+\mu_{F} \omega_{\eta} M}, \\
b_{p_{t} n}^{\text {mean }}=b_{p_{t} n}^{\text {corr } f}=\frac{p_{0} \omega_{\eta}\left(\frac{3}{2} \frac{S_{1 / 4}}{S_{1 / 2}}-\frac{M S_{3 / 4}}{\left(S_{1 / 2}\right)^{2}}\right)}{4 \omega_{\mu} S_{1 / 2}+M \mu_{F} \omega_{\eta}},
\end{gathered}
$$




$$
\begin{gathered}
b_{p_{t} p_{t}}^{\text {mean }}=b_{p_{t} p_{t}}^{\text {corf }}= \\
=\frac{\omega_{\eta} \mu_{F}\left(9 S_{1 / 2}^{3}-12 S_{1 / 4} S_{3 / 4} S_{1 / 2}+4 M S_{3 / 4}^{2}\right)}{16 \gamma S_{1} S_{1 / 2}^{2}+\omega_{\eta} \mu_{F}\left(9 S_{1 / 2}^{3}-12 S_{1 / 4} S_{3 / 4} S_{1 / 2}+4 M S_{3 / 4}^{2}\right)+16 \omega_{\mu} S_{1 / 2}\left(S_{1} S_{1 / 2}-S_{3 / 4}^{2}\right)},
\end{gathered}
$$

where

$$
S_{v}=\sum_{i=1}^{M} \bar{\eta}_{i}^{v}
$$

The coincidence of the asymptotes, obtained by two methods, was not obvious in advance, since for a nonlinear correlation function these two alternative definitions of the correlation coefficients, used in these methods, are not identical and they can generally lead to different results [18].

As an example we consider the non-uniform string distribution, were in a part $m$ of the cells the mean strings density is $a$ times larger, than in the rest $(1-m) M$ cells:

$$
\begin{aligned}
& M_{+}=m M \quad \bar{\eta}_{+}=a \bar{\eta} \\
& M-M_{+}=(1-m) M \quad \bar{\eta}
\end{aligned}
$$

By formulae (18) and (19) we see that the correlation coefficient between mean transverse momentum and multiplicity is connected with the correlation coefficient between multiplicities:

$$
b_{p_{t} n}=\frac{p_{0} \omega_{\eta}\left(\frac{3}{2} \frac{S_{1 / 4}}{S_{1 / 2}}-\frac{M S_{3 / 4}}{\left(S_{1 / 2}\right)^{2}}\right)}{4 \omega_{\mu} S_{1 / 2}+M \mu_{F} \omega_{\eta}}=b_{n n} \frac{p_{0} S_{3 / 4}}{\mu_{B}\left(S_{1 / 2}\right)^{2}}\left(\frac{3}{2} \frac{S_{1 / 4} S_{1 / 2}}{M S_{3 / 4}}-1\right) .
$$

The correlation coefficient between multiplicities is always positive, but the bracket in this relation can be negative. In this case the correlation coefficient between mean transverse momentum and multiplicity will be also negative.

$$
\frac{3}{2} \frac{S_{1 / 4} S_{1 / 2}}{M S_{3 / 4}}-1<0
$$

This expression can be rewritten in following form:

$$
\begin{gathered}
m^{2}\left(3 a^{3 / 4}-3 a^{1 / 2}-3 a^{1 / 4}+3\right)+m\left(-2 a^{3 / 4}+3 a^{1 / 2}+3 a^{1 / 4}-4\right)+1<0, \\
F(a, m) \equiv m^{2}\left(3 a^{3 / 4}-3 a^{1 / 2}-3 a^{1 / 4}+3\right)+m\left(-2 a^{3 / 4}+3 a^{1 / 2}+3 a^{1 / 4}-4\right)+1 .
\end{gathered}
$$

In Fig. 2 we can see that there are such non-uniform distributions of strings for which this coefficient becomes negative.

For the correlation coefficient $b_{p_{t} p_{t}}$ its dependence on the scaled variance of the number of particles produced from a single string was analyzed (Fig. 3). We see that this dependence disappears in the case of the uniform string distribution, as one can expect from [15].

The obtained asymptotes for the correlation coefficient between transverse momenta $b_{p_{t}} p_{t}$ were compared with the results of the MC numerical calculations of this coefficient (Fig. 4). In this figure we see that the asymptotic regime is reached at string density corresponding to $\bar{\eta} \gtrsim 6$. 


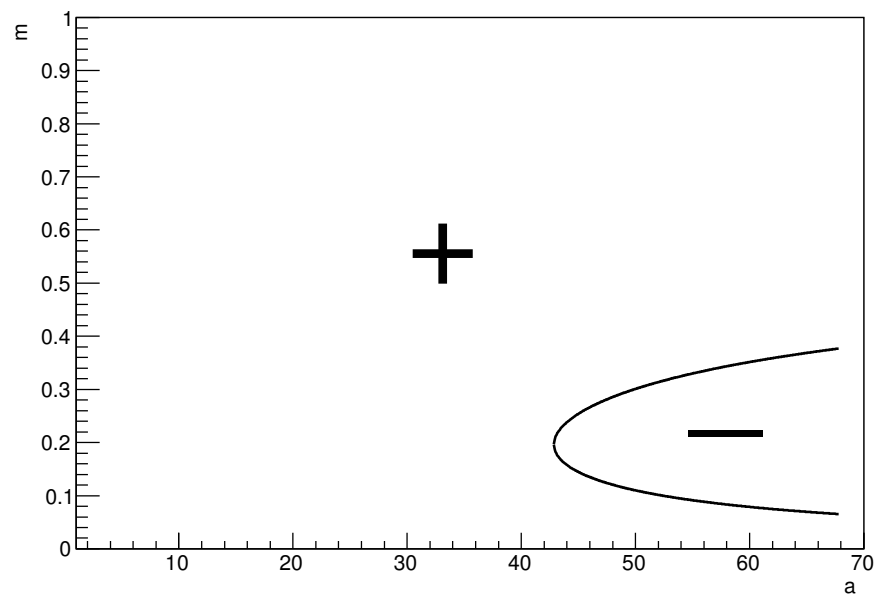

Figure 2. The region of the parameters $a$ and $m$ (see definitions in the text), where the correlation coefficient between mean transverse momentum and multiplicity is negative.

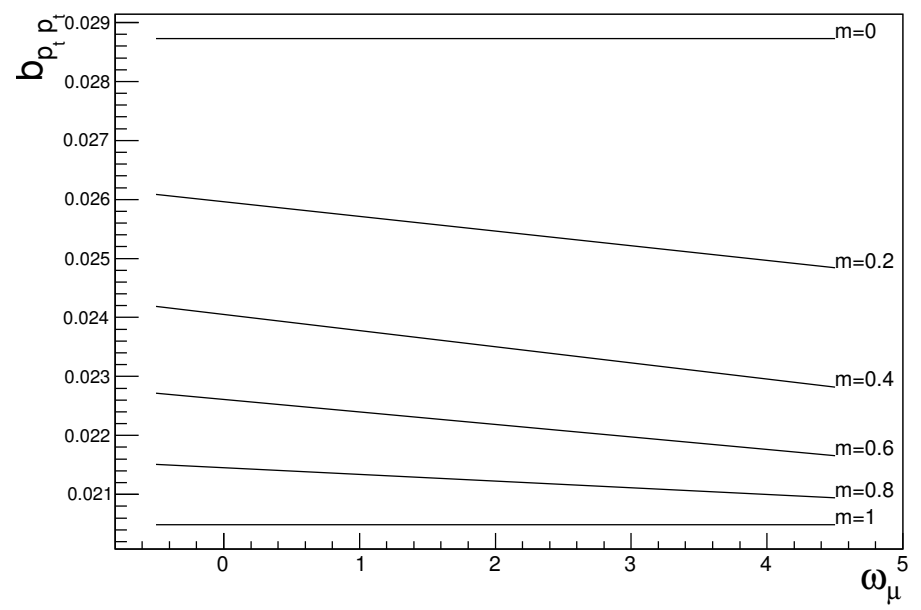

Figure 3. The dependence of correlation coefficient $b_{p_{t} p_{t}}$ on the scaled variance of the number of particles produced from a single string

\section{Conclusion}

The long-range correlations between mean transverse momentum and multiplicity $\left(p_{t}-n\right)$ and between mean transverse momenta $\left(p_{t}-p_{t}\right)$ of charged particles produced in high energy hadronic collisions in separated rapidity intervals are studied in the model with quark-gluon strings (color flux tubes) as sources [1-4]. The effects of the string interaction and fusion [5-8] are taken into account in the simplified form by implementing a finite lattice (grid) in the impact parameter plane [9-11]. 


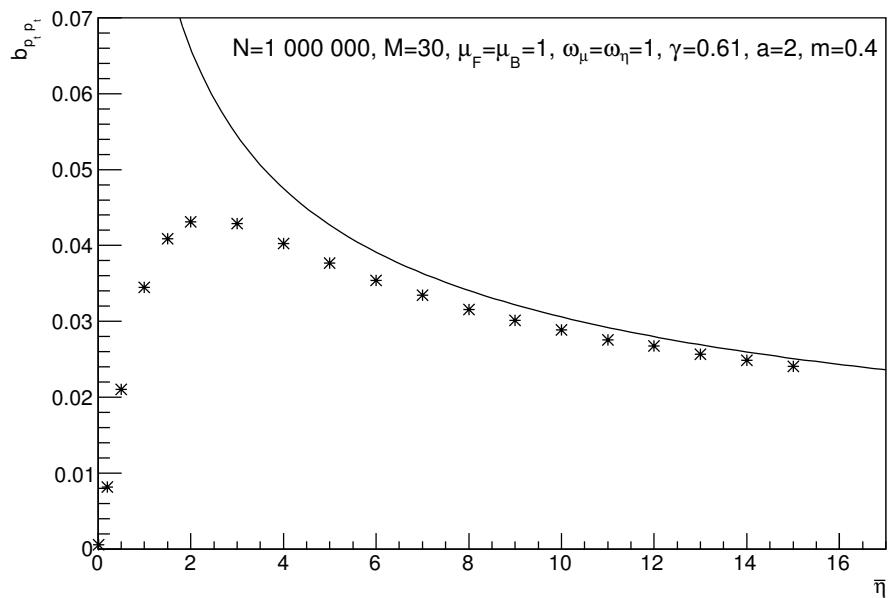

Figure 4. The results of the MC numerical calculations (dots) and the received analytical asymptotes (line) for the correlation coefficient $b_{p_{t} p_{t}}$ for the different values of string density $\bar{\eta}=\frac{\sum_{k=1}^{M} \bar{\eta}_{k}}{M}$

In the framework of the model the analytical expressions for the asymptotes of the correlation coefficients at large non-uniform string density were obtained by two very different methods basing on the alternative definitions of the correlation coefficients. It was shown that in the leading approximation the asymptotes of the correlation coefficients obtained by these two methods coincide. The obtained results enable to understand qualitatively the main features of the long-range correlation behavior at large string density. Thus, it was found the strong dependence of the $p_{t}-n$ correlation coefficient on the inhomogeneity of the string distribution in contrast to the $n-n$ correlation between multiplicities. In particular it was shown that there are such distributions of strings for which the $p_{t}-n$ correlation coefficient becomes negative.

For the $p_{t}-p_{t}$ correlation coefficient the dependence on the scaled variance of the number of particles produced from a single string is analyzed. It is shown that the dependence on this parameter disappears for a homogeneous string distribution, as it was expected in [15]. The analytical asymptotes of the correlation coefficients obtained at large string density are compared with the results of the MC numerical calculations of these coefficients. This enables to evaluate the string density at which the asymptotic regime is reached and also to check both the analytic asymptotic formulas and the MC numerical algorithms, which then can be applied for the calculations of the correlation coefficients in a non-asymptotic region at small string density.

\section{Acknowledgement}

The research was funded by the SPbGU outgoing academic mobility grant and by the grant of the COST Action "Theory of hot matter and relativistic heavy-ion collisions" (THOR).

\section{References}

[1] A.B.Kaidalov, Phys. Lett. B 116:6, 459-463 (1982) 
[2] A.B.Kaidalov, K.A.Ter-Martirosyan, Phys. Lett. B, 117, 247 (1982)

[3] A.Capella, U.Sukhatme, Chung-I Tan, J.Tran Thanh Van, Phys. Lett. B 81:1, 68-74 (1979)

[4] A.Capella,U.Sukhatme, Chung-I Tan, J. Tran Thanh Van, Phys. Rep. 236, 225 (1994)

[5] T.S. Biro, H.B. Nielsen, J. Knoll, Nucl. Phys. B 245, 449 (1984)

[6] A. Bialas, W. Czyz, Nucl. Phys. B 267, 242 (1986)

[7] M.A. Braun, C. Pajares, Phys. Lett. B 287, 154 (1992)

[8] M.A. Braun, C. Pajares, Nucl. Phys. B 390, 542 (1993)

[9] V.V. Vechernin and R.S. Kolevatov, Vestn. Peterb. Univ. Ser. 4: Fiz. Khim., No. 2, 12-23 (2004); arXiv:hep- ph/0304295v1

[10] V.V. Vechernin and R.S. Kolevatov, Vestn. Peterb. Univ. Ser. 4: Fiz. Khim., No. 2, 11-27 (2004); arXiv:hep- ph/0305136v1

[11] M.A. Braun, R.S. Kolevatov, C. Pajares, V.V. Vechernin, Eur. Phys. J. C 32:4, 535-546 (2004); arXiv: hep-ph/0307056

[12] V.V. Vechernin, R.S. Kolevatov, Phys. Atom. Nucl. 70, 1797 (2007)

[13] M.A.Braun, C.Pajares, V.V.Vechernin, Nucl. Phys. A 906, 14 (2013)

[14] M.A. Braun, C. Pajares, V.V. Vechernin, Eur. Phys. J. A 51, 44 (2015)

[15] V.V. Vechernin, Theor.Math.Phys. 184, 1271 (2015); ibid. 190, 251 (2017).

[16] V. Vechernin, Nucl. Phys. A 939, 21-45 (2015)

[17] M.A. Braun, C. Pajares, V.V. Vechernin, Phys. Lett. B 493:1-2, 54-64 (2000)

[18] V.V. Vechernin, Proceedings of the Baldin ISHEPP XX, vol. 2, JINR, Dubna, p.10 (2011); arXiv:1012.0214 